\title{
One night in San Marino
}

\author{
Petr Benda ${ }^{1,2}$ \& Marcel Uhrin ${ }^{3}$
}

\author{
${ }^{1}$ Department of Zoology, National Museum (Natural History), Václavské nám. 68, CZ-110 00 Praha 1, \\ Czech Republic; e-mail: petr.benda@nm.cz \\ ${ }^{2}$ Department of Zoology, Charles University in Prague, Viničná 7, CZ-128 44 Praha 2, Czech Republic \\ ${ }^{3}$ Department of Zoology, Institute of Biology and Ecology, P. J. Šafárik University, Šrobárova 2, \\ SK-041 54 Košice, Slovakia
}

Benda P. \& Uhrin M., 2020: One night in San Marino. - Journal of the National Museum (Prague), Natural History Series 189: 163-166.

\begin{abstract}
During several hours of acoustic survey of bats in Borgo Maggiore, San Marino, on 30 August 2020, foraging activity of at least six bat species was recorded. Five of them (Eptesicus serotinus, Hypsugo savii, Pipistrellus pipistrellus, P. kuhlii, and Tadarida teniotis) are common and widespread across the Apennine Peninsula and are already known members of the bat fauna of San Marino. The sixth species, Nyctalus noctula, is here reported from this country for the first time and the bat fauna of San Marino increases to 18 species.
\end{abstract}

Key words: bats, echolocation, acoustic survey, distribution, new records

Received: December 7, 2020 | Accepted: December 11, 2020 | Published on-line: December 16, 2020

The Republic of San Marino is a tiny nation in the Apennine Peninsula, typical by its small area $\left(61 \mathrm{~km}^{2}\right.$, the third smallest in Europe) combined with a relatively steep altitudinal gradient (53-756 $\mathrm{m}$ a. s. l.) and a rather high population density (ca. 570 people $/ \mathrm{km}^{2}$, the fourth highest in Europe). A tourist can explore such a small territory during one or two days. Since San Marino is a country rich in historic monuments, it is a good destination for a short stopover during a journey along the eastern shore of Italy, being less than $10 \mathrm{~km}$ from the Adriatic A14 highway.

During a way back from a bat research trip to Greece in the summer of 2020, the first author of this contribution made a one-day rest stop in San Marino that included an overnight stay. He used this opportunity to make also a brief survey of flight activity of bats in this small country. In the first half of the night, he tried to detect bat echolocation calls in the centre of Borgo Maggiore (Piazza Grande; 4356'22”N, 12²6'47”E; $520 \mathrm{~m}$ a. s. l.); this small town is full of historic buildings and adjacent to the steep and forested northern slope of Monte Titano (756 m a. s. l.), the highest point of the country and a seat of the country capital Città di San Marino. 


\begin{tabular}{|c|c|c|c|c|c|c|c|}
\hline species & n1 & n2 & $\begin{array}{c}\text { SF } \\
{[\mathrm{kHz}]}\end{array}$ & $\begin{array}{l}\text { Fmax } \\
{[\mathrm{kHz}]}\end{array}$ & $\begin{array}{c}\text { EF } \\
{[\mathrm{kHz}]}\end{array}$ & $\begin{array}{c}\text { D } \\
{[\mathrm{ms}]}\end{array}$ & $\begin{array}{c}\text { IPI } \\
{[\mathrm{ms}]}\end{array}$ \\
\hline \multirow[t]{2}{*}{ Eptesicus serotinus } & 7 & 20 & $31.3 \pm 2.3$ & $29.1 \pm 1.5$ & $29.1 \pm 1.9$ & $8.4 \pm 4.0$ & $202.4 \pm 22.5$ \\
\hline & & & $24.7-35.1$ & $24.7-32.3$ & $24.1-32.3$ & $3.3-15.8$ & $170.0-252.0$ \\
\hline \multirow[t]{2}{*}{ Hypsugo savii } & 44 & 38 & $35.2 \pm 1.8$ & $34.1 \pm 1.0$ & $33.3 \pm 0.7$ & $8.4 \pm 2.8$ & $157.9 \pm 44.5$ \\
\hline & & & $32.6-41.5$ & $32.0-36.6$ & $31.4-35.1$ & $4.6-17.0$ & $69.0-214.0$ \\
\hline \multirow[t]{2}{*}{ Pipistrellus pipistrellus } & 9 & 38 & $51.2 \pm 4.7$ & $47.7 \pm 1.5$ & $47.6 \pm 1.3$ & $5.8 \pm 2.1$ & $136.0 \pm 48.2$ \\
\hline & & & $44.5-64.4$ & $44.2-53.4$ & $44.5-51.9$ & $2.0-11.1$ & $84.0-250.0$ \\
\hline \multirow[t]{2}{*}{ Pipistrellus kuhlii } & 29 & 37 & $43.4 \pm 4.9$ & $38.6 \pm 1.1$ & $38.0 \pm 1.2$ & $6.7 \pm 3.6$ & $113.2 \pm 39.5$ \\
\hline & & & $37.5-28.0$ & $36.3-41.2$ & $36.0-41.5$ & $1.3-19.7$ & $75.0-227.0$ \\
\hline \multirow[t]{2}{*}{ Nyctalus noctula } & 5 & 8 & $22.1 \pm 0.7$ & $21.5 \pm 0.6$ & $21.4 \pm 0.7$ & $12.3 \pm 3.8$ & $719.0 \pm 166.9$ \\
\hline & & & $20.7-22.9$ & $20.4-22.3$ & $20.4-22.3$ & $6.6-16.4$ & $622.0-968.0$ \\
\hline Tadarida teniotis & 1 & - & - & - & - & - & - \\
\hline
\end{tabular}

Table 1. Results of the acoustic bat survey in Borgo Maggiore, San Marino, on 30 August 2020, and its analysis. Abbreviation explanations: $\mathrm{n} 1=$ total number of sequences identified; $\mathrm{n} 2=$ number of call pulses analysed; $\mathrm{SF}=$ start frequency; $\mathrm{Fmax}=$ frequency of maximum energy; $\mathrm{EF}=$ end frequency; $\mathrm{D}=$ call duration; $\mathrm{IPI}$ = inter-pulse interval; upper lines: mean \pm standard deviation; lower lines = value ranges. The recorded quality of the call sequence of Tadarida teniotis was poor for parameter measurings, but sufficient for species identification.

The bat calls were recorded with the help of the automatic ultrasound detector Batlogger M (Elekon AG, Switzerland) in the period between the sunset (ca. 20.05 CEST) and the CEST midnight on 30 August 2020. The recorded calls were analysed in BatExplorer 2.0 software (Elekon AG, Switzerland), using call characteristics known from the respective geographical area (Russo \& Jones 2002). Altogether, 115 noise sequences were recorded, 99 of them were identified as sequences of bat calls, and 95 sequences were assigned to a particular bat species (Table 1).

This small research effort revealed a fairly good foraging activity of at least six bat species at and in the surroundings of the Piazza Grande (Table 1), the area combining urban and natural conditions and with street lamp illumination which also contributed to the site attractiveness for bats. Five of the recorded bat species rank among common inhabitants of the Apennine Peninsula, namely Eptesicus serotinus (Schreber, 1774), Hypsugo savii (Bonaparte, 1837), Pipistrellus pipistrellus (Schreber, 1774), P. kuhlii (Kuhl, 1817), and Tadarida teniotis (Rafinesque, 1814). Moreover, $H$. savii and P. kuhlii, two most typical Mediterranean urban elements among the species, covered more than three quarters of the recorded call activity (76.8\%). This situation is quite common and corresponds to a usual experience from southern Europe (Russo \& Jones 2003, Campedelli et al. 2013) and these five bat species are already known members of the Sammarinese bat fauna (Scaravelli 2012).

On the other hand, the evidence of Nyctalus noctula (Schreber, 1774) is less usual in the Appenine Peninsula, this species is only rarely found in the peninsular part of Italy and has not yet been recorded from San Marino (Lanza 2012, Anonymous 2016). The calls of $N$. noctula were detected five times during 2.5 hours, the sequences were recorded in the intervals of 13-73 minutes (mean $37.8 \mathrm{~min}$ ) - this corresponds to five particular passes (21:14, $21: 27,22: 03,22: 32,23: 45)$. The bat or bats foraged perhaps in a broader area that surrounds Monte Titano.

This migratory species is expected to be present in the southernmost sections of Europe only during its seasonal migrations and cold parts of a year, while the summer occurrence 
and supposed reproduction of N. noctula in Italy was reported only from the northern parts of the country (Lapini et al. 2019). Its occurrence in San Marino in late August could be regarded exceptional, although it may be perhaps linked to the beginning of autumn migration. However, rather abundant records of $N$. noctula have been recently reported from the mountainous areas west of San Marino (Campedelli et al. 2013, Ducci et al. 2019).

The bat fauna of San Marino still seems to be very insufficiently known. It started to be studied relatively late, in the 1990s; the review by Scaravelli \& Bassi (1994) presented only four bat species from the country. The first survey of bats of San Marino (Scaravelli et al. 2001) yielded confirmed occurrence of six species. After a specialised long-term research, Scaravelli (2012) reported fourteen bat species in the country. Most recently, an official report (Anonymus 2016) provided a list of seventeen species of the Sammarinese bat fauna, although without any reference to the original records. Anyway, no species of the genus Nyctalus has been known from San Marino till now. With the recent discovery of N. noctula, the number of species in the bat fauna of this small country increases to eighteen.

Anyway, this number still represents roughly just a half of the number reported for the continental bat fauna of Italy, which comprises 34 species (Lanza 2012, Ancillotto et al. 2019). Thus, the research of bats of San Marino is still not finished; perhaps many more nights are necessary to be spent in various parts of this country until its nightlife is completely described.

\section{Acknowledgements}

This contribution was supported by the Ministry of Culture of the Czech Republic (\# DKRVO 2019-2023/6.IX.b, 00023272) and by the Scientific Grant Agency of the Slovak Republic $(1 / 0298 / 19)$.

\section{References}

Ancillotto L., Mori E., Bosso L., Agnelli P. \& Russo D., 2019: The Balkan long-eared bat (Plecotus kolombatovici) occurs in Italy - first confirmed record and potential distribution. Mammalian Biology 96: 61-67. https://doi.org/10.1016/j.mambio.2019.03.014

Anonymous, 2016: Convenzione sulla Diversità Biologica. V Rapporto Nazionale. - San Marino: Dipartimento Territorio Ambiente, $38 \mathrm{pp}$.

Campedelli T., Londi G., Cutini S. \& Florenzano G. T., 2013: Il popolamento di chirotteri di un'area dell'Appennino tosco-romagnolo (Comuni di Pieve S. Stefano e Badia Tedalda, Arezzo) (Mammalia Chiroptera). - Quaderno di Studi e Notizie di Storia Naturale della Romagna 37: 123-140.

Ducci L., Roscioni F., Carranza M. L., Agnelli P., Russo D., Frate L., Loy A., Santini G. \& Di Febbraro M., 2019: The role of protected areas in preserving habitat and functional connectivity for mobile flying vertebrates: the common noctule bat (Nyctalus noctula) in Tuscany (Italy) as a case study. - Biodiversity and Conservation 28: 1569-1592.

Lanza B., 2012: Fauna d'Italia. Mammalia V. Chiroptera. - Milano: Calderini-Edizioni Calderini, 786 pp.

Lapini L., Dorigo L., Luca M., Lapia M., Bufo P. \& Urso G., 2019: Remarks about some noteworthy bats from northeastern Italy (Friuli Venezia Giuila Region: Chiroptera: Vespertilionidae: Myotis bechsteinii, Myotis capaccinii, Myotis daubentonii, Nyctalus lasiopterus, Nyctalus noctula). - Quaderni del Museo Civico di Storia Naturale di Ferrara 7: 91-100.

Russo D. \& Jones G., 2002: Identification of twenty-two bat species (Mammalia: Chiroptera) from Italy by analysis of time-expanded recordings of echolocation calls. - Journal of Zoology, London 258: 91-103. https://doi.org/10.1017/s0952836902001231

Russo D. \& Jones G., 2003: Use of foraging habitats by bats in a Mediterranean area determined by acoustic surveys: conservation implications. - Ecography 26: 197-209. https://doi. org/10.1034/j.1600-0587.2003.03422.x 
Scaravelli D., 2012: Chirotteri della Repubblica di San Marino. Rapporto 2010-2011. - Scritti, Studi e Ricerche di Storia Naturale della Repubblica di San Marino 3: 103-111.

Scaravelli D. \& Bassi S., 1994: Indagini su chirotteri nell'Appennino romagnolo-marchigiano. - Biogeographia 17: 547-552. https://doi.org/10.21426/b617110415

Scaravelli D., Suzzi Valli A., Cecchetti A. \& Casali S., 2001: First account on bats of S. Marino Republic. - In: Wołoszyn B. W. (ed.): Bats \& Man. Million Years of Coexistence. Proceedings of the VIIIth EEBRS. Vol. II. Distribution, Ecology, Paleontology and Systematics of Bats. P. 231. Kraków: Institute of Systematics and Evolution of Anoimals PAS. 